\title{
Effects of Chronic Kidney Disease on 5-Year Follow- Up Clinical Outcomes After Off-pump Coronary Artery Bypass Grafting-A Retrospective Study
}

Xihui Li ( $\sim$ heart2000@163.com )

Peking University First Hospital https://orcid.org/0000-0003-0268-2386

\section{Siyu Zhang}

Peking University First Hospital

Feng Xiao

Peking University First Hospital

\section{Research article}

Keywords: chronic kidney disease, coronary artery bypass grafting, off-pump, follow-up

Posted Date: January 23rd, 2021

DOI: https://doi.org/10.21203/rs.3.rs-151058/v1

License: (c) (i) This work is licensed under a Creative Commons Attribution 4.0 International License.

Read Full License 


\section{Abstract}

\section{Backgroud}

To investigate the effect of chronic kidney disease (CKD) on mid- and long-term clinical outcomes after off-pump coronary artery bypass grafting (CABG).

\section{Methods}

This was a retrospective analysis of data of 1141 discharged patients from January 2010 to June 2018 after undergoing off-pump CABG. Follow-up endpoints included stroke, myocardial infarction, heart failure, revascularization, and all-cause death.Patients with preoperative estimated glomerular filtration rate calculated, using the Chronic Kidney Disease Epidemiology equation of $\geq 60 \mathrm{~mL} / \mathrm{min} / 1.73 \mathrm{~m}^{2}$ and < $60 \mathrm{~mL} / \mathrm{min} / 1.73 \mathrm{~m}^{2}$, were assigned tonormal Group (1, 910 cases) and CKD group (231 cases), respectively. The effects of CKD on selected endpoint events were compared and analyzed.

Results

There was a higher proportion of women, more preoperative complications, and a higher incidence of early postoperative complications in patients with CKD than inthose with normal renal function.After 1-9 years of follow-up (mean5.0 \pm 2.2 years), the incidences of stroke, myocardial infarction, and all-cause mortality were significantly higher in the CKD than in the normal renal function group, whereas incidences of revascularization and heart failure were not.Logistic regression analysis showed that preoperative CKD was a risk factor for stroke, myocardial infarction, and all-cause death during follow-up. After correctingfor common confounding factors, such as sex, age, and left ventricular ejection fraction, preoperative CKD was a risk factor for myocardial infarction and all-cause death.

Conclusions

In patientsundergoing off-pump CABG, preoperative CKD is associated with increases in the incidences of myocardial infarction and all-cause mortality 5 -year postoperation..

\section{Background}

Chronic kidney disease (CKD) is a major risk factor for coronary artery disease (CAD) [1], andtherisk of death, cardiovascular events, and hospitalization increasewith decreasingestimated glomerular filtration rate (EGFR)[2].Coronary artery bypass grafting(CABG) and percutaneous coronary intervention ( $\mathrm{PCl})$ are effective treatments for severe coronary heart disease. A meta-analysis found that CABG is associated withsignificantly lowerlong-term mortality in patients with CKD and end-stage renal diseasethan is $\mathrm{PCI}$ [3].

Whether off-pump or on-pump CABG is preferable for patients with coronary heart disease with CKDis controversial. Current studies have shown that off-pumpCABG may be superior, possibly because offpump obviates the need forcardiopulmonary bypass and protectsrenal function in the perioperative 
period. It is alsoassociated with fewer complications,including allogeneic blood transfusion, postoperative thoracotomy hemostasis, acute kidney injury, and respiratory system dysfunction [4].In particular, off-pump CABG significantly reduces operative mortality and risk of postoperative dialysis in patients with moderate or severe renal insufficiency [5]. Off-pump CABG is currently preferred in mainland China, accounting for approximately $95 \%$ of total CABG procedures in our department.Management of such patients is very complicated, and the impact of the comorbidity of CKD on mid- and long-term clinical outcomes of off-pump CABG hasrarelybeen studied. We therefore conducted a retrospective study of 5-year(1-9 years) follow-up outcomes of 1141 patients, who had undergone off-pump CABG from January 2010 to June 2018 in the Department of Cardiac Surgery, Peking University First Hospital.

\section{Methods}

Inclusion criteria were as follows: consecutive, first-time patients undergoing off-pump CABGdischarged from our hospital from January 2010 to June 2018. Exclusion criteria were as follows: acute renal insufficiency, preoperative end-stage renal disease dependent ondialysis, intraoperative transfer to onpump, death within 30 days after surgery, incomplete data, and refusal to attend for follow-up.The Chronic Kidney Disease Epidemiology Collaboration (CKD-EPI) equation was used to calculate eGFR[6].Patients with eGFR $\mathrm{CKD}_{\text {EPII }}>60 \mathrm{~mL} / \mathrm{min} / 1.73 \mathrm{~m}^{2}$ were classified as having normal renal functionand those with $<60 \mathrm{~mL} / \mathrm{min} / 1.73 \mathrm{~m}^{2}$ as havingCKD.Baseline and operative data during hospitalization andpostoperative outpatient or telephone follow-up data were collected for statistical analysis.Clinical outcomes included stroke, myocardial infarction, heart failure, revascularization, and allcause death.

SPSS21.0 software(IBM Corp., Armonk, NY, USA) was used for analysis,the $\chi^{2}$ or Fisher's exact test for analyzing count data between two groups and agroup $t$-test for measurement data. The effect of preoperative CKD on all clinical outcome events during follow-up was analyzed by logistic regression.Odds ratios (ORs) and 95\% confidence intervals were calculated byunivariateanalysis and corrected for common confounding factors, such as sex, age, and left ventricular ejection fraction (LVEF). $P<0.05$ was consideredto denote a significant difference (two-sided test).

\section{Results}

A total of 1141 patients were included in the study: 910 in the normal Group and 231 in the CKD group.Comparisons of baseline and operative data during hospitalization are shown in Table 1.There was a higher proportion of women, older mean age, and higher incidence of preoperative and postoperative complications in the CKD group than in the normal renal function group.

All patients underwentoutpatient or telephone follow-up for 1-9 years (mean $5.0 \pm 2.2$ years). The incidences of stroke, myocardial infarction, and all-cause mortality were significantly higher in the CKD than the normal renal function group, whereas the incidences of revascularization and heart failure were not. Clinical endpoints according to study group areshown in Table 2. 
Results of logistic regression analysis on the effect of preoperative CKD on clinical outcome events areshown in Table 3.Preoperative CKD was found to be a risk factor for stroke, myocardial infarction, and all-cause mortality. After correctionfor common confounding factors, such as sex, age, and LVEF, preoperative CKD was identified asa risk factor for myocardial infarction and all-cause mortality.

\section{Discussion}

Patients with CKD have a significantly increased risk of coronary heart disease, not only because oftraditionally recognized CAD risk factors such as diabetes and hypertension, but also because of other non-traditionally recognized cardiovascular disease risk factors associated with uremia, including inflammation, oxidative stress, and abnormal calcium-phosphorus metabolism[1].Lipid metabolism disorders, mainly hypertriglyceridemia, are risk factors for coronary heart diseasein patients with CKD.This accounts for a prevalence of coronary heart disease of approximately $12 \%$ in patients with Stage 3 or greater CKD, compared with only $5 \%$ in those with normal renal function [7].CKD is often accompanied by disorders of mineral bone metabolism, hypocalcemia,hyperphosphatemia, and secondary hyperparathyroidism, all of which canaccelerate systemic atherosclerosis and vascular calcification [8].Inflammatory responses, oxidative stress, impaired endothelial cell function, coronary artery calcification, hyperhomocysteinemia, immunosuppression, and other mechanismscan contribute to significant acceleration of atherosclerosis, leading to a poor prognosis in this patient group.Complicationsof coronary heart disease, such as myocardial infarction and heart failure, are the main causes of death [9].Particularly in dialysis-dependent patients withadvanced CKD, the death rate is as high as 40\%-50\% [10].A 2012 epidemiological survey reported anincidence of CKD in mainland China of $10.8 \%$ and estimated that there were 119.5 million patients withCKD in that country[11].Increasing numbers of thesepatients will require $C A B G$ in the future. There is an urgent need to improvepostoperative long-term survival rate.

In patients with severe coronary heart disease and non-dialysis-requiring CKD, CABG achieves significantly lowermortality, re-infarction, and revascularization rates than do $\mathrm{PCl}$ and drug therapy [12, 13].In one study 2108 patients with CKD who underwentPCl usingdrug-eluting stents, $(n=1165)$ orCABG $(n=943)$ were prospectively analyzed in a nonrandomized study with anaverage follow-up of 41.4 months.Although no significant differences in all-cause death, stroke, and myocardial infarction were observed between the two groups, the revascularization rate was significantly higher in the PCl group [14].A meta-analysis of 29,246 patients enrolled in 11 studies showed that CABG achievedlower long-term all-cause mortality, cardiac mortality, andincidences of myocardial infarction and revascularization, and fewer major adverse cardiacand cerebrovascular events (MACCE) than did drug-eluting stents forrevascularization in patients with multivesselcoronary heart disease and CKD. However, mortality and risk of cerebrovascular eventshaverecentlyincreased [15].Although CABG can significantly improve the survival rate, it also increases the incidence of postoperativerenal failure, especially in high-risk patients [16]. 
Off-pump CABG reportedly has a protective effect on renal function in the perioperative period because of it does not entailcardiopulmonary bypass; it also reduces the incidences of complications, such as allogeneic blood transfusion, post-operative thoracotomy hemostasis, acute kidney injury, and respiratory system dysfunction [4].However, in the CORONARY study, no significant difference was observedbetweenon-pump and off-pump CABGin effectson renal function at the 1 year follow-up, and offpump had no demonstrable long-term renal protective effect [17]. In the present study, there was a higher proportion of women, older mean age, and higher incidences of preoperative and postoperative complications in theCKD thanin the normal group.The follow-up period ranged from 1 to 9 years, with an average of 5.0 \pm 2.2 years. The incidences of stroke, myocardial infarction, and all-cause mortality were significantly higher in the CKD than in the normal renal function group; however, those of revascularization and heart failure were not.Logistic regression analysis showed that preoperative CKD was a risk factor for stroke, myocardial infarction, and all-cause mortality. After correctingfor common confounding factors such as sex, age, and LVEF, preoperative CKD was identified as a risk factor for myocardial infarction and all-cause mortality in the off-pump CABG over a mid- to long-term follow-up.

CKD and end-stage renal disease not only increase the risk of CAD, but also change its clinical manifestations and main symptoms.Management of patients with CAD and CKD is complexbecause of the possibility of complications and adverse effects during interventions[1].Protection of renal function and improvement in postoperative survival are criticalfor discharged patients with CKD not undergoing dialysiswho have undergone CABG.There is a need for cooperation among cardiac surgeons, cardiologists, nephrologists, and endocrinologists to enhance postoperative management and constructcomprehensive strategies, to improve prognosis and reduce complications and mortalityin highrisk patients.

First, the identification of high-risk patients facilitates rational allocation of medical resources, targeted prevention, and management.As renal function worsens, the length of hospital stay and medical costsand the number of complications increase.Decreasesin creatinine clearance to 80,60 , and $20 \mathrm{~mL} / \mathrm{min}$ reportedly lead to cost increases of $10 \%, 20 \%$, and $30 \%$, respectively.Additionally, the incidence of need for dialysis and mortality increase postoperatively[18].Grouping of CKD severity on the basis ofeGFR calculated by the CKD-EPI equation effectively predictspostoperative complications and mortality in both the short-and long-term [23]. eGFR $\mathrm{CKD}_{\text {-EPI }}<30 \mathrm{~mL} / \mathrm{min} / 1.73 \mathrm{~m}^{2},<41 \mathrm{~mL} / \mathrm{min} / 1.73 \mathrm{~m}^{2},<27 \mathrm{~mL} / \mathrm{min} / 1.73$ $\mathrm{m}^{2}$, and $<29 \mathrm{~mL} / \mathrm{min} / 1.73 \mathrm{~m}^{2}$ are predictors of early mortality, stroke, prolonged length of hospital stay, and postoperative dialysis substitution, respectively.Additionally, eGFR $\mathrm{CKD}_{\text {EPI }}<26 \mathrm{~mL} / \mathrm{min} / 1.73 \mathrm{~m}^{2}$, $<25 \mathrm{~mL} / \mathrm{min} / 1.73 \mathrm{~m}^{2},<35 \mathrm{~mL} / \mathrm{min} / 1.73 \mathrm{~m}^{2}$, and $<29 \mathrm{~mL} / \mathrm{min} / 1.73 \mathrm{~m}^{2}$ are predictors of all-cause death, cardiovascular death, myocardial infarction, and heart failure,respectively, during follow-up (average follow-up time 66 months) [19].Therefore, the management of high-risk patients needs to be further strengthened.

Second, active treatment of comorbidities such as hypertension, diabetes, and anemia is vital.Hypertension and diabetes are the two commonestcomorbidities in patients with 
CKD.Recommendations for treatment of diabetic nephropathy include control of $\mathrm{HB} 1 \mathrm{Ac}<7 \%$, blood pressure $<140 / 90 \mathrm{mmHg}$, and treatment with renin-angiotensin system blockers [20].Patients with CKD have a high incidence of hypertension that may lead to or originate from CKD.Hypertension aggravates CKD, resulting in uncontrollable hypertension [21].The 2017 American College of Cardiology/American Heart Association Hypertension Guidelines set a BP target of $<130 / 80 \mathrm{~mm} \mathrm{Hg}$ for patients with CKD and other strongcardiovascular risk factors toreduce mortality in patients with CKD. However, fewdata are available for patients with diabetes or Stage 4-5 CKD [22].Anemia, another common manifestation of renal insufficiency, causes decreased erythropoietin secretion and renal anemia.Anemia predicts the risk of cardiovascular events and death in patients with coronary heart disease [23].

Third, measures for secondary prevention of coronary heart disease should be actively continued postoperatively, including oral antiplatelet drugs, statin treatment, and good living habits.

\section{Conclusions}

Patients withCKD undergoing CABG are older preoperatively, have more complications, and moreearly postoperative complications than those with normal renal function.5-year follow-up after CABGrevealed associations between preoperative CKD and higher incidences of $\mathrm{MI}$ and all-cause mortality (after correcting forcommon confounding factors such as sex, age, and LVEF).Among patients with CKD undergoing off-pump $C A B G$, it is necessary to identify high-risk patients and strengthen comprehensive management to reduce complications and improve long-term survival.

\section{Abbreviations}

chronic kidney disease CKD

coronary artery bypass grafting CABG

coronary artery disease CAD

estimated glomerular filtration rate EGFR

percutaneous coronary intervention $\mathrm{PCl}$

left ventricular ejection fraction LVEF

Chronic Kidney Disease Epidemiology Collaboration CKD-EPI

major adverse cardiacand cerebrovascular events MACCE

\section{Declarations}




\section{Acknowledgments}

We thank Dr Trish Reynolds, MBBS, FRACP, from LiwenBianji, Edanz Group China (www.liwenbianji.cn/ac), for editing the English text of a draft of this manuscript.

\section{Authors' Contributions}

Siyu Zhangand XihuiLi Literature search; Feng Xiao and SiyuzhangData collection.. All authors read and approved the finalmanuscript.

\section{Competing interests}

The authors have no conflict of interest to declare.

\section{Funding}

We received no funding for this study.

\section{Availability of data and materials}

The datasets used and/or analysed during the current study are available from the corresponding author on reasonable request.

\section{Ethics Approval and consent to participate}

This study was approved by the biomedical research ethics committee of Peking University First Hospital (approval No. 2018, scientific research 242). And consent was waived.

\section{Consent for Publication}

Not applicable

\section{References}

1. Sarnak, M. J., Amann, K., Bangalore, S., et al.. Chronic kidney disease and coronary artery disease: JACC state-of-the-art review. J. Am. Coll. Cardiol. 2019;74: 1823-1838.

2. Go AS, Chertow GM, Fan D, et al. Chronic kidney disease and the risks of death, cardiovascular events, and hospitalization, N Engl J Med , 2004『351ه1296-1305

3. Kannan A, Poongkunran C, Medina R, et al. Coronary Revascularization in Chronic and End-Stage Renal Disease: A Systematic Review and Meta-analysis. Am J Ther.2016;23:e16-28.

4. Lamy A, Devereaux PJ, Prabhakaran D, et al. Off-pump or on-pump coronary-artery bypass grafting at 30 days. N Engl J Med.2012;366:1489-97.

5. Ueki C, Miyata H, Motomura N, et al. Off-pump technique reduces surgical mortality after elective coronary artery bypass grafting in patients with preoperative renal failure[J]. The Journal of Thoracic 
and Cardiovascular Surgery, 2018;156:976-983.

6. Michels WM,Grootendorst DC, Verduijn M, et al. Performance of the Cockcroft-Gault, MDRD, and new CKD-EPI formulas in relation to GFR, age, and body size. Clin J Am Soc Nephrol. 2010;5:1003-1009.

7. Lamprea-Montealegre JA, Mcclelland RL, Grams M, et al. Coronary heart disease risk associated with the dyslipidaemia of chronic kidney disease[J]. Heart, 2018; 104:1455-1460.

8. Reiss AB囚 Miyawaki N囚Moon J囚et al. CKD, arterial calcification, atherosclerosis and bone health: Inter-relationships and controversies. Atherosclerosis. 2018;278:49-59

9. Cai Q, Mukku VK, Ahmad M. Coronary Artery Disease in Patients with Chronic Kidney Disease: A Clinical Update. Current Cardiology Reviews, 2013;9:331-339

10. Collins AJ, Foley RN, Herzog C, et al. US Renal Data System 2012 Annual Data Report. Am J Kidney Dis. 2013; 61:A7, e1-476.

11. Zhang L,Wang F,Wang L,et al. Prevalence of chronic kidney disease in China: a cross-sectional survey.Lancet,2012; 379:815-822.

12. Roberts JK, Rao SV, Shaw LK, et al. Comparative Efficacy of Coronary Revascularization Procedures for Multivessel Coronary Artery Disease in Patients with Chronic Kidney Disease『Am J Cardiol. 2017; 119: 1344-1351.

13. Charytan DM, Desai M, Mathur M, et al.Reduced risk of myocardial infarct and revascularization following coronary artery bypass grafting compared with percutaneous coronary intervention in patients with chronic kidney disease. Kidney Int. 2016;90:411-421.

14. Se Hun Kang, Cheol Whan Lee, Sung-Cheol Yun, et al. Coronary Artery Bypass Grafting vs. DrugEluting Stent Implantation for Multivessel Disease in Patients with Chronic Kidney Disease. Korean Circ J. 2017; 47: 354-360

15. Wang $Y$, Zhu S , Gao P , et al. Comparison of coronary artery bypass grafting and drug-eluting stents in patients with chronic kidney disease and multivessel disease: A meta-analysis[J]. European Journal of Internal Medicine, Eur J Intern Med. 2017;43:28-35.

16. Charytan DM, Natwick T, Solid CA, et al. Comparative Effectiveness of Medical Therapy, Percutaneous Revascularization, and Surgical Coronary Revascularization in Cardiovascular Risk Subgroups of Patients With CKD: A Retrospective Cohort Study of Medicare Beneficiaries. Am J Kidney Dis. 2019 ; 74:463-473.

17. Garg AX, Devereaux PJ, Yusuf S, et al. Kidney function after off-pump or on-pump coronary artery bypass graft surgery: a randomized clinical trial. JAMA. 2014;311:2191-8.

18. LaPar DJ, Rich JB, Isbell JM, et al. Preoperative Renal Function Predicts Hospital Costs and Length of Stay in Coronary Artery Bypass Grafting. Ann Thorac Surg. 2016;101:606-612

19. Gelsomino S, Del Pace SD, Parise O, et al. Impact of renal function impairment assessed by CKDEPI estimated glomerular filtration rate on early and late outcomes after coronary artery bypass grafting. International Journal of Cardiology.2017;227:778-787 
20. 20.Umanath K, Lewis JB Update on Diabetic Nephropathy: Core Curriculum 2018 Am J Kidney Dis. 2018;71:884-895

21. Horowitz B, Miskulin D, Zager P. Epidemiology of hypertension in CKD.Adv Chronic Kidney Dis. 2015;22:88-95.

22. ChangAR, Lóser M, MalhotraR, et al. Blood Pressure Goals in Patients With CKD: A Review of Evidence and Guidelines. Clin J Am Soc Nephrol.2019;14:161-169.

23. Cook JR ,Dillie KS , Hakeem A, et al. Effectiveness of Anemia and Chronic Kidney Disease as Predictors for Presence and Severity of Coronary Artery Disease in Patients Undergoing Stress Myocardial Perfusion Study[J]. American Journal of Cardiology, 2008, 102:266-271.

\section{Tables}

Table 1. Comparison of baseline and operative data between the CKD and normal groups

\begin{tabular}{|c|c|c|c|c|}
\hline & $\begin{array}{c}\text { normalGroup } 1 \\
(\mathrm{n}=910)\end{array}$ & $\begin{array}{c}\text { CKDGroup } 2 \\
(n=231)\end{array}$ & $t / X^{2}$ & $p$ \\
\hline Age(ys) & $63.4 \pm 9.2$ & $69.7 \pm 8.9$ & 9.328 & 0.000 \\
\hline Female sex $(\%)$ & $228(25.1)$ & $111(48.1)$ & 46.656 & 0.000 \\
\hline Hypertension( $\%$ ) & $595(65.4)$ & $194(84.0)$ & 29.871 & 0.00 \\
\hline Diabetes(\%) & $347(38.1)$ & $120(51.9)$ & 14.546 & 0.000 \\
\hline \multicolumn{2}{|c|}{ Stroke before operation(\%)90(21.1) } & $67(28.5)$ & 6.970 & 0.008 \\
\hline Hyperlipemia(\%) & $384(42.1)$ & $113(47.4)$ & 3.384 & 0.066 \\
\hline OMI(\%) & $345(37.9)$ & $92(39.8)$ & 0.286 & 0.593 \\
\hline $\operatorname{LVEF}(\%)$ & $61.2 \pm 13.3$ & $57.8 \pm 15.0$ & 3.380 & 0.001 \\
\hline Hemoglobin $(\mathrm{g} / \mathrm{L})$ & $135.1 \pm 15.4$ & $123.1 \pm 17.9$ & 10.211 & 0.000 \\
\hline AF before operation $(\%)$ & $40(4.3)$ & $21(8.4)$ & 6.704 & 0.01 \\
\hline $\mathrm{BNP}(\mathrm{pg} / \mathrm{mL})$ & $243 \pm 415$ & $428 \pm 625$ & 4.270 & 0.000 \\
\hline Left main involved(\%) & $246(27.0)$ & $78(33.8)$ & 4.108 & 0.043 \\
\hline Emergency(\%) & $17(1.9)$ & $4(1.7)$ & - & 1.000 \\
\hline LIMA use(\%) & $788(86.6)$ & $169(73.2)$ & 25.693 & 0.000 \\
\hline $\mathrm{LCOS}$ & $58(6.4)$ & $22(9.5)$ & 2.804 & 0.094 \\
\hline IABP use & $58(6.4)$ & $30(13.0)$ & 11.321 & 0.001 \\
\hline Grafts & $2.7 \pm 0.8$ & $2.5 \pm 0.8$ & 2.143 & 0.032 \\
\hline Re-open & $8(0.9)$ & $4(1.7)$ & - & 0.276 \\
\hline $\operatorname{Re}$ intubation & $10(1.1)$ & $7(3.0)$ & 4.682 & 0.030 \\
\hline $\mathrm{AKI}$ & $239(26.3)$ & $102(44.2)$ & 28.146 & 0.000 \\
\hline ARDS & $90(9.9)$ & $29(12.6)$ & 1.400 & 0.237 \\
\hline Stroke peri-operative & $13(14.3)$ & $9(3.9)$ & 5.932 & 0.015 \\
\hline UGH & $12(13.2)$ & $9(3.9)$ & 6.774 & 0.029 \\
\hline AFperi-operative & $153(16.8)$ & $52(22.5)$ & 4.058 & 0.044 \\
\hline
\end{tabular}

AF: atrialfibrillation; AKI: acute renal injury; ARDS:acute respiratory distress syndrome; BNP: brain natriuretic peptide; COPD:chronic obstructive pulmonary disease; IABP: intra-aortic balloon pump; LCOS: 
low cardiac output syndrome; LIMA: left internal mammary artery; LVEF:left ventricular ejection fraction; OMI: old myocardial infarction; UGH:upper gastrointestinal hemorrhage.

Table 2. Comparison of clinical outcome events between CKD and normal groups

\begin{tabular}{|c|c|c|c|c|}
\hline $\begin{array}{l}\text { Normal Group CKDGroup } 2 t \\
(\mathrm{n}=910) \quad(\mathrm{n}=231)\end{array}$ & & & & \\
\hline Stroke(\%) & $52(5.7)$ & $22(9.5)$ & 4.408 & 0.036 \\
\hline $12(1.3)$ & $9(3.9)$ & 6.774 & & \\
\hline Revascularization(\%) & $37(4.1)$ & $12(5.2)$ & 0.571 & 0.450 \\
\hline Heart failure(\%) & $14(1.5)$ & $4(1.7)$ & -- & 0.771 \\
\hline All-cause mortality( $\%)$ & $50(5.5)$ & $27(11.7)$ & 11.231 & 0.001 \\
\hline
\end{tabular}

Ml: myocardial infarction

Table 3. Logistic regression analysis of the effect of CKD on clinical outcome events (before and after adjusted)

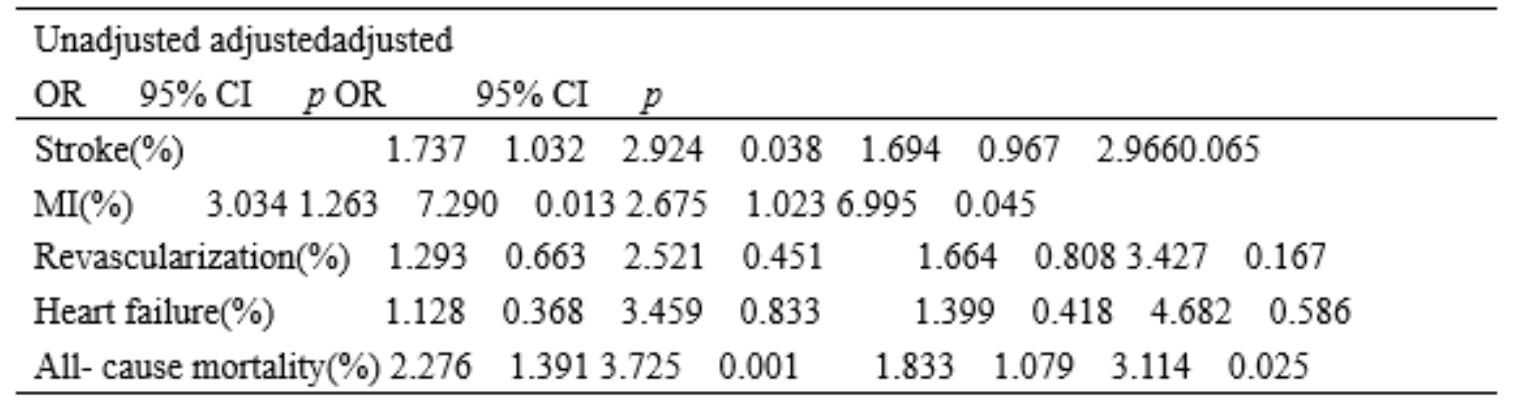

Ml: myocardial infarction 